\title{
Gemeinsam Wissen schaffen. Vernetzte Beiträge von wissenschaftlichen Bibliotheken und Wiki-Communitys für eine digitale Landeskunde
}

\section{Digitale Landeskunde in Sachsen: die Rolle der SLUB Dresden}

Als sächsische Landesbibliothek sammelt die SLUB Dresden bzw. ihre Vorgängereinrichtungen seit Ende des 18. Jahrhunderts systematisch Literatur aus und über Sachsen - so genannte Saxonica. Zunächst auf Literatur zur sächsischen Geschichte konzentriert, begann sich der Saxonica-Begriff bereits im 19. Jahrhundert auf andere Wissenschaftsgebiete mit regionalem Bezug wie die Naturund die Volkskunde, die Geografie, die Altertumskunde oder die Sprachforschung auszudehnen. Heute umfasst er Publikationen aller Medienarten analog wie digital - und Wissensgebiete, die sich inhaltlich auf das Gebiet Sachsens in seinen jeweiligen Grenzen beziehen. Die SLUB ist damit ein zentraler Dienstleister für (professionelle und ehrenamtliche) Forschungstätigkeiten auf dem Gebiet der Geschichte, Kultur und Landeskunde im Sinn einer interdisziplinären Regionalforschung ${ }^{1}$ und ein wichtiger Akteur bei dem Anliegen, den Wandel von der Papier- zur digitalen Bibliothek zu gestalten. ${ }^{2}$

Mitte der 2000er Jahre begann die SLUB, systematisch landes- und regionalkundlich relevante Quellen zu digitalisieren. ${ }^{3}$ Zunächst stellte sie zentrale

1 Vgl. Mannsfeld, Karl: Landeskunde als interdisziplinäre Regionalforschung. In: Denkströme. Journal der Sächsischen Akademie der Wissenschaften 6 (2011). S. 56-60. http://www. denkstroeme.de/heft-6/s_56-60_mannsfeld. - Der vorliegende Beitrag beruht auf einer Präsentation zur WikiCon in Leipzig am 9. September 2017. Ich danke meinen Kollegen Jens Bemme und Thomas Stern für regelmäßigen Ideenaustausch zum Thema. Der Titel des Beitrags ist einem Videoclip der Helmholtz-Gemeinschaft zur Erläuterung des Citizen-Science-Konzepts entlehnt, vgl. https://www.youtube.com/watch?v=49Ho7ut-DhI. Alle aufgeführten Internetressourcen wurden zuletzt am 30. November 2017 aufgerufen.

$2 \mathrm{Zu}$ diesem Wandlungsprozess vgl. z. B. Schade, Frauke: Praxishandbuch Digitale Bibliotheksdienstleistungen. Strategie und Technik der Markenkommunikation. Berlin, Boston: de Gruyter 2016, bes. S. 15-25. https://doi.org/10.1515/9783110346558.

3 Vgl. jetzt Meyer, Julia u. Martin Munke: Digitale Landeskunde für Sachsen. Programme und Projekte an der SLUB Dresden. In: Bibliotheksdienst 52 (2018) H. 2. S. 106-119. https://doi.org/ 10.1515/bd-2018-0015.

๖ Open Access. () 2018 Martin Munke, publiziert von De Gruyter. (@) BY-NC-ND Dieses Werk ist lizenziert unter der Creative Commons Attribution-NonCommercial-NoDerivatives 4.0 Lizenz. 
wissenschaftliche Zeitschriften online und frei zugänglich zur Verfügung, darunter das (Neue) Archiv für sächsische Geschichte und die Dresdner Hefte. Zeitschrift zur Regional- und Kulturgeschichte Dresdens, außerdem die Mitteilungen des Landesvereins Sächsischer Heimatschutz aus dem Bereich der Heimatzeitschriften mit wissenschaftlichem Anspruch. Die Saxonica in den Digitalen Sammlungen der Bibliothek sind momentan ${ }^{4}$ mit mehr als 19000 Titeln in etwa 40000 Bänden die umfassendste Kollektion im digitalen Bestand. Über die Retrodigitalisierung hinaus, die durch das von der SLUB koordinierte „Landesdigitalisierungsprogramm für Wissenschaft und Kultur des Freistaates Sachsen“ (LDP) intensive finanzielle Förderung erfährt, ${ }^{5}$ hat die Bibliothek allein und gemeinsam mit Partnern verschiedene Projekte realisiert, die durch Erschließung und Kontextualisierung von Digitalisaten eine Reihe von Mehrwerten für die landeskundliche Forschung in Sachsen bieten. ${ }^{6}$ Dazu zählen etwa das „Virtuelle Kartenforum 2.0"7 als Webanwendung zur Georeferenzierung und zum Vergleich digitalisierter Karten, das Portal „Historische Adressbücher“8 ${ }^{\text {zur sei- }}$ tengenauen Recherche nach Straßen- und Personennamen von mehr als 210 sächsischen Städten und Gemeinden bis 1945 oder das Erschließungs- und Digitalisierungsvorhaben zur NS-Tageszeitung Der Freiheitskampf ${ }^{9}$ - Projekte, von deren weiterführenden Anwendungsmöglichkeiten auch verschiedene WikiCommunitys im Bereich der deutschsprachigen Wikipedia ${ }^{10}$ und des Schwesterprojekts Wikisource, ${ }^{11}$ aber auch regionale Initiativen wie z. B. das Stadtwiki Dresden ${ }^{12}$ profitieren können.

4 Stand vom 4. Dezember 2017.

5 Vgl. ausführlich Bonte, Achim: Aus Sachsen in die Welt - das sächsische Landesdigitalisierungsprogramm. In: Bibliothek der Zukunft - Zukunft der Bibliothek. Festschrift für Elmar Mittler. Hrsg. von Andreas Degkwitz. Berlin, Boston: de Gruyter 2016. S. 10-23. https://doi. org/10.1515/9783110464016-003.

6 Vgl. jeweils mit Angaben zu den beteiligten Partnern und weiteren Literaturhinweisen Meyer/Munke, Digitale Landeskunde (wie Anm. 3), S. 111-116.

$7 \mathrm{Vgl}$. https://kartenforum.slub-dresden.de.

8 Vgl. https://adressbuecher.sachsendigital.de.

9 Vgl. http://slubdd.de/freiheitskampf.

10 Vgl. https://de.wikipedia.org.

11 Vgl. https://de.wikisource.org.

12 Vgl. http://www.stadtwikidd.de. 


\section{Wissenschaft und Wikipedia: „Ignorieren zwecklos ${ }^{\text {“13 }}$}

Mehr als 2,1 Millionen Artikel und in den vergangenen Jahren durchgängig in den Top Ten der meist frequentierten Internetseiten in Deutschland und weltweit $^{14}$ - das Onlinelexikon Wikipedia ist im Internet für viele eine der ersten Anlaufadressen zur Information über und weiteren Recherche zu Themen aus allen Wissensgebieten. Die Bedeutung als Instrument der Wissensvermittlung und -generierung wird auch an den folgenden Zahlen aus den Jahren 2014/2015 deutlich: „An jedem beliebigen Tag benutzen 10,5 \% aller Deutschen ab 14 Jahren die Wikipedia. [...] $42 \%$ aller Jugendlichen (12 bis 19 Jahre alt) in Deutschland lesen regelmäßig Wikipedia, $11 \%$ täglich. [...] $94 \%$ der MitarbeiterInnen deutscher Hochschulen und Universitäten nutzen Wikipedia beruflich. ${ }^{\text {"15 Trotz }}$ aller Vorbehalte im akademischen Kontext ist die Wikipedia damit eines der wirkmächtigsten Beispiele des Citizen-Science-Konzepts, das die Bedeutung bürgerwissenschaftlichen Engagements im Kontext des digitalen Wandels hervorhebt: Jeder darf, zumindest in der Theorie, an der Wissensgenerierung und -vermittlung teilhaben. ${ }^{16}$ Ein weiteres Ziel der von den Bibliotheken prominent vertretenen Open-Science-Philosophie, also den wissenschaftlichen Prozess in allen seinen Teilaspekten frei im Internet zugänglich zu machen, wird von der Wikipedia eingelöst: der Open-Access-Gedanke, die freie Verfügbarmachung von (wissenschaftlicher) Literatur bzw. im Wikipedia-Kontext der zentralen Befunde aus dieser Literatur im Internet. ${ }^{17}$ Studien haben dabei gezeigt, dass neben frei zugänglichen Materialien besonders Artikel aus Fachzeitschriften mit einem hohen Impact-Faktor überdurchschnittlich häufig in Wikipedia-Artikeln

13 König, René: Wissenschaft und Wikipedia - eine „Zwangsehe“ mit Hindernissen? In: Wikipedia. Ein kritischer Standpunkt. 24.-26. September 2010, Leipzig. http://www.cpov.de/?p=79. 14 Zahlen nach https://de.wikipedia.org/wiki/Wikipedia:Statistik.

15 Vgl. Heller, Lambert: Von der Gatekeeper-Insel raus auf den Ozean des freien Wissens. In: TIB-Blog. Weblog der Technischen Informationsbibliothek (TIB), 28. November 2016. http:// blogs.tib.eu/wp/tib/2016/11/28/von-der-gatekeeper-insel-raus-auf-den-ozean-des-freien-wissens/.

16 Zu Begriff und Konzept(en) vgl. Finke, Peter: Citizen Science. Das unterschätzte Wissen der Laien. München: Oekom 2014.

$17 \mathrm{Zu}$ den Zusammenhängen vgl. einführend Herb, Ulrich: Offenheit und wissenschaftliche Werke. Open Access, Open Review, Open Metrics, Open Science \& Open Knowledge. In: Open Initiatives. Offenheit in der digitalen Welt und Wissenschaft. Hrsg. von Ulrich Herb. Saarbrücken: universaar 2012. S. 11-44, bes. S. 11-19 u. 37-38. https://doi.org/10.22028/D291-25636. Zu den entsprechenden Aktivitäten an der SLUB vgl. https://www.slub-dresden.de/open-science/. 
zitiert werden und somit eine weit größere Verbreitung erfahren, als es allein im akademischen Kontext der Fall wäre. ${ }^{18}$

Das Verhältnis zwischen professionellen und Bürgerwissenschaften war und ist in der Wikipedia gleichwohl ambivalent. ${ }^{19}$ Das kollaborative Vorgehen, Schreiben ohne Klarnamen und die Annahme einer fehlenden redaktionellen Überarbeitung, die allerdings zumindest teilweise im Kontext von so genannten Themenportalen versucht wird, werden einerseits als mangelnder Qualitätsausweis gesehen. Anderseits spielen, bedingt durch das hohe Ranking bei Google, Wikipedia-Artikel schon seit Längerem eine kaum zu überschätzende Rolle bei der thematischen Erstinformation durch Studenten für universitäre Lehrveranstaltungen. ${ }^{20}$ Und trotz aller Vorbehalte ist ja auch der weit überwiegende Teil der Lehrkräfte, wie oben gezeigt, beruflich zumindest gelegentlich in der Wikipedia aktiv. Insgesamt zeigt sich in den vergangenen Jahren eine im englischsprachigen Kontext schon etwas länger zu beobachtende zunehmende Bereitschaft, sich aktiv mit dem Phänomen „Wikipedia“ auseinanderzusetzen, anstatt die Artikelqualität pauschal zu verdammen und bei sich im Kreis drehenden Diskussionen zur Zitierfähigkeit stehenzubleiben. Dies äußert sich etwa in der wachsenden Anzahl von Qualifikationsarbeiten zum Thema, aber auch in der (in Deutschland noch sehr seltenen) Möglichkeit für Studenten, WikipediaArtikel als Teil universitärer Prüfungsleistungen $\mathrm{zu}$ verfassen. ${ }^{21}$

18 Vgl. Schmeja, Stefan: Wikipedia und Open Access: gut füreinander, gut für die Gesellschaft. In: TIB-Blog. Weblog der Technischen Informationsbibliothek (TIB), 20. Oktober 2015. https:// blogs.tib.eu/wp/tib/2015/10/20/wikipedia-und-open-access-gut-fuereiander-gut-fuer-die-gesellschaft/. Ausführlich vgl. Teplitskiy, Misha, Grace Lu u. Eamon Duede: Amplifying the Impact of Open Access. Wikipedia and the Diffusion of Science. In: Journal of the Association for Information Science and Technology 68 (2017) H. 9. S. 2116-2127. https://doi.org/10.1002/ asi.23687.

19 Als umfassende Bestandsaufnahme am Beispiel meiner „Heimatdisziplin“ - der Geschichtswissenschaft - vgl. Wozniak, Thomas, Jürgen Nemitz u. Uwe Rohwedder (Hrsg.): Wikipedia und Geschichtswissenschaft. Berlin, Boston: de Gruyter 2015. https://doi.org/10.1515/ 9783110376357. Einführend Wozniak, Thomas: Zehn Jahre Berührungsängste: Geschichtswissenschaft und Wikipedia. Eine Bestandsaufnahme. In: Zeitschrift für Geschichtswissenschaft 60 (2012) H. 3. S. 247-264.

20 Hübner, Hans-Jürgen: Qualität in der Wikipedia. Binnenperspektive eines Historikers. In: Wikipedia und Geschichtswissenschaft (wie Anm. 19). S. 185-204, hier S. 185-186. https://doi. org/10.1515/9783110376357-014.

21 Vgl. Wozniak, Berührungsängste (wie Anm. 19), S. 259-261. Für die angloamerikanische Wissenschaft vgl. z. B. Knight, Charles u. Sam Pryke: Wikipedia and the University, a case study. In: Teaching in Higher Education 17 (2012) H. 6. S. 649-659. https://doi.org/10.1080/ 13562517.2012.666734; Bayliss, Gemma: Exploring the Cautionary Attitude Toward Wikipedia in Higher Education. Implications for Higher Education Institutions. In: New Review of Academic Librarianship 19 (2013) H. 1. S. 36-57. https://doi.org/10.1080/13614533.2012.740439. 
Neben der Wahrnehmung durch die akademische ist hier ebenso die Selbstwahrnehmung der Wikipedia-Community zu hinterfragen. Bei näherem Hinsehen wird der mit dem Citizen-Science-Gedanken verbundene partizipative Ansatz nur in engen Grenzen umgesetzt. 2008 waren es nämlich nur knapp $3 \%$ der Nutzer, die selbst Inhalte beitrugen und nicht nur als Rezipienten auftraten. ${ }^{22}$ Seit 2007 sinkt die Anzahl der Beiträger beständig, zumal das sich immer weiter ausdifferenzierende Regelwerk den Einstieg häufig erschwert und die so genannten Relevanzkriterien einerseits ein Mittel der Qualitätssicherung sein sollen, andererseits so gerade in der deutschsprachigen Wikipedia eine zusätzliche Hürde aufgebaut wird. ${ }^{23}$ Selbst von den mehr als zwei Millionen Benutzerkonten, für die sich die Nutzer aktiv registrieren müssen, waren nur etwa 20000 regelmäßig schreibend tätig. ${ }^{24}$ Zudem weisen besonders die kollaborativ erstellten Artikel eher Defizite in Struktur, Prägnanz und Verständlichkeit auf als von einem Autor allein verfasste Lemmata. ${ }^{25}$ Der Befund, dass mehr als ein Viertel der Artikelersteller und -bearbeiter nach eigenen Angaben über einen akademischen Abschluss verfügt, kann dadurch relativiert werden, dass dieser Abschluss nicht in der Disziplin erworben worden sein muss, in der der betreffende Artikel angesiedelt ist. So kann der professionelle Wissenschaftler argumentativ zum Bürgerwissenschaftler werden. Gleichzeitig gerät damit ein weiterer Aspekt des Citizen-Science-Konzepts in den Blick, nämlich die Vermittlung von Wissen in gesellschaftlich relevanten Feldern - z. B. zu Natur- und Umweltthemen, im geisteswissenschaftlichen Bereich etwa zum kritischen Umgang mit historischen Themen, die schließlich von aktueller politischer Relevanz sein können. ${ }^{26}$ Gerade im regionalen und lokalen Kontext, im konkreten Lebensumfeld vor Ort sind solche Überlegungen die Hauptmotivation, in Citizen-ScienceProjekten wie der Wikipedia, aber auch ganz klassisch im Verein aktiv zu werden. ${ }^{27}$

22 Pscheida, Daniela: Das Wikipedia-Universum. Wie das Internet unsere Wissenskultur verändert. Bielefeld: transcript 2010, S. 375. https://doi.org/10.14361/transcript.9783839415610. 23 Dijk, Ziko van: Die Wikipedia - das ideale Hilfsmittel für den Einstieg in ein historisches Thema? In: Wikipedia und Geschichtswissenschaft (wie Anm. 19). S. 1-14, hier S. 13. https:// doi.org/10.1515/9783110376357-003; Wozniak, Berührungsängste (wie Anm. 19), S. 248-252. Am Punkt der Relevanzkriterien setzen Regional- und Stadtwikis an, in denen weit kleinteiliger vorgegangen wird.

24 Vgl. Hübner, Qualität (wie Anm. 20), S. 190.

25 Vgl. Dijk, Wikipedia (wie Anm. 23), S. 7-8.

26 Vgl. Hübner, Qualität (wie Anm. 20), S. 203-204.

27 Vgl. Finke, Citizen Science (wie Anm. 16), S. 126-149. 


\section{Das Personen.Wiki der SLUB: gemeinsame Arbeit von Bibliothekaren und Wiki-Nutzern}

Ein Beispiel für diesen regionalspezifischen Ansatz ist das zwischen 2006 und 2016 von der SLUB betriebene Personen.Wiki. ${ }^{28}$ Es entstand als „Nebenprodukt“ der (regional-)bibliografischen Arbeit im Kontext der Sächsischen Bibliografie ${ }^{29}$ und des „Fachkatalogs Technikgeschichte“. ${ }^{30}$ Die dabei gesammelten Daten vor allem Name und Alternativnamen, Geburts- und Sterbeort und -datum dienen im Rahmen der Sacherschließung der Individualisierung von Personen und beziehen sich oft auf solche von „nur“ lokaler und/oder regionaler Bedeutung, die entsprechend in übergeordneten biografischen Datenbanken wie der (Neuen) Deutschen Biographie ${ }^{31}$ aus Relevanzgründen keine Aufnahme finden. Weiterhin werden hier auch Informationen über lebende Personen erfasst, die in den meisten anderen biografischen Nachschlagewerken nicht berücksichtigt werden.

Zentrales Instrumentarium zur Verzeichnung solcher Daten ist die Gemeinsame Normdatei (GND), die von der Deutschen Nationalbibliothek (DNB), den deutschen Bibliotheksverbünden und anderen Einrichtungen gemeinsam geführt wird. ${ }^{32}$ Vor zehn Jahren war der Bestand an Datensätzen besonders zu

28 Vgl. Letocha, Michael: Das Personen-Wiki der Sächsischen Landesbibliothek - Staats- und Universitätsbibliothek Dresden. Ein Nebenprodukt regionalbibliografischer Arbeit als Arbeitsund Auskunftsmittel. In: Biografische Lexika im Internet. Internationale Tagung der „Sächsischen Biografie“ in Dresden (30. und 31. Mai 2008). Hrsg. von Martina Schattkowsky u. Frank Metasch. Dresden: Thelem 2009. S. 157-165, hier S. 157-158.

29 Siehe dazu unten, Kap. 4.

30 http://swb.bsz-bw.de/DB=2.305. Die technikhistorische Bibliografie wurde seit 1961 als „Informationsdienst Geschichte der Technik“, seit 1971 als „Bibliographie Geschichte der Technik“ und seit 1992 schließlich als „Fachkatalog Technikgeschichte“ an der SL(U)B geführt, seit 1998 im Kontext des Sondersammelgebiets Technikgeschichte der Deutschen Forschungsgemeinschaft (DFG). Zum 1. Januar 2016 wurde dieses Sondersammelgebiet an die Bibliothek des Deutschen Museums in München übergeben, die es im Rahmen des „Fachinformationsdienstes Geschichtswissenschaft" weiter betreuen wird.

31 https://www.deutsche-biographie.de. Vgl. einführend Ebneth, Bernhard: Die Neue Deutsche Biographie (NDB). Vom Lexikon zum Online-Informationssystem. In: Archiv und Wirtschaft 45 (2012) H. 2. S. 76-84.

32 Vgl. einführend Wiechmann, Brigitte: Normdaten. In: Praxishandbuch Bibliotheksmanagement. Hrsg. von Rolf Griebel, Hildegard Schäffler u. Konstanze Söllner. Berlin, Boston: de Gruyter 2014. S. 380-391, bes. S. 382-384. https://doi.org/10.1515/9783110303261.380. Die Bedeutung im Kontext bio-bibliografischen Arbeitens skizziert Ebneth, Bernhard: Auf dem Weg $\mathrm{zu}$ einem historisch-biographischen Informationssystem. Datenintegration und Einsatz von Normdaten am Beispiel der Deutschen Biographie und des Biographie-Portals. In: Jahrbuch für Universitätsgeschichte 16 (2013). S. 261-290. 
Personen der hier skizzierten Kategorie in der Personennamendatei (PND), einem der Vorläufer der GND, noch um ein Vielfaches geringer. Zudem waren (und sind teilweise bis heute) bei Persönlichkeiten von überregionaler Bedeutung deren lokale und regionale Bezüge, die im Kontext des regionalbibliografischen Arbeitens besonders interessant sind, im Datensatz zumeist nicht erfasst. Um diese Aspekte auszugleichen, etablierten die Bibliografen der SLUB das Personen.Wiki auf Basis der MediaWiki-Software ${ }^{33}$ als eine offene Datenbank, an der auch Bibliothekskollegen und Nichtbibliotheksmitarbeiter mitwirken konnten. ${ }^{34}$ Deren Ergänzungen wurden vor der Freischaltung redaktionell gesichtet. Neben den genannten biografischen Eckdaten fanden ebenso kurze Biogramme zu wichtigen Lebensstationen Aufnahme, ergänzt um weiterführende Links und Literaturverweise.

Bis 2016 entstanden so in Zusammenarbeit von Bibliothekaren und interessierten Nutzern fast 20000 Datensätze mit teilweise nur hier verfügbaren Angaben zu Personen des öffentlichen Lebens im Freistaat und im historischen Sachsen. Aufgrund der technischen Entscheidung seitens der SLUB, keine eigenen MediaWiki-Instanzen mehr zu betreiben, wurde auch das Personen.Wiki zum 31. Oktober 2016 zunächst abgeschaltet. Die noch nicht in der GND erfassten Personen werden dort durch die Mitarbeiter des Saxonica-Referats nach und nach angelegt. ${ }^{35}$ Über das gemeinnützige „Internet Archive“ ist eine archivierte Version des Wikis nach wie vor erreichbar. ${ }^{36}$ Gleichzeitig wurde der komplette Datenbestand an einen ehrenamtlichen Mitarbeiter des Stadtwikis Dresden übergeben, der eine zunächst noch nicht frei zugängliche neue Instanz des Wikis aufgesetzt hat. Die Freischaltung dieser Version ist in Vorbereitung, sodass die Betreuung und Weiterführung dieses regional- und landeskundlichen Auskunftsinstrumentes aus der Bibliothek an die Wiki-Community übergeben werden soll.

33 https://www.mediawiki.org.

34 Vgl. Letocha, Personen-Wiki (wie Anm. 28), S. 162-165.

35 Vgl. Munke, Martin: Neue Wege bei Personendaten. Personen.Wiki der SLUB wird in Gemeinsame Normdatei GND überführt. In: SLUBlog, 7. Oktober 2016. https://blog.slub-dresden. de/beitrag/2016/10/07/neue-wege-bei-personendaten-personenwiki-der-slub-wird-in-gemeinsame-normdatei-gnd-ueberfuehrt/.

36 https://web.archive.org/web/20161006075219/http://personen-wiki.slub-dresden.de/. 


\section{Verknüpfung durch Normdaten: Sächsische Bibliografie online und Artikel in der Wikipedia}

Zentrales Nachweisinstrument für die regional- und landeskundliche Literatur über Sachsen ist die Sächsische Bibliografie. ${ }^{37}$ Seit 1992 erfolgt deren laufende Verzeichnung datenbankgestützt. 1998 wurde die Bibliografie im Internet abrufbar. Die Retrokonversion und die Einarbeitung der Druckausgaben in die Sächsische Bibliografie online sind vollständig erfolgt, sodass hier die seit Beginn des 20. Jahrhunderts systematisch erfasste Literatur recherchierbar ist. Die verzeichneten Publikationen werden über eine Systematik auf Basis der Regensburger Verbundklassifikation (RVK) und über normierte Schlagworte sachlich erschlossen. ${ }^{38}$

Die in der Bibliografie erfasste Literatur ist von hoher Relevanz nicht nur für die professionelle Forschung zur sächsischen Regional- und Landeskunde, sondern auch und gerade für ehrenamtlich Tätige, wie sie in der Wikipedia und in Regiowikis aktiv sind. Hoch spezialisierte Fragestellungen und lokale Besonderheiten, die an keiner Universität behandelt werden, erfahren hier ihre Berechtigung und werden von enthusiastischen Heimatforschern untersucht. Zum Ausbau der Vernetzung von Onlineangeboten erfolgt seit einiger Zeit unter anderem für Personenartikel in der Wikipedia eine Verknüpfung mit dem zugehörigen Normdatum der GND, das auch in der Bibliografie zur eindeutigen Identifizierung von Personen verwendet wird. Über so genannte BEACONDateien lassen sich Verlinkungen $\mathrm{zu}$ anderen Onlineangeboten herstellen, die ihrerseits mit Normdaten auf GND-Basis arbeiten. ${ }^{39}$ Um die gegenseitige Verknüpfung zu unterstützen und die Identifikation relevanter Titel zu erleichtern, stellte das Referat Saxonica der SLUB im September 2016 eine Vorlage zur leichten Einbindung von Daten aus der Bibliografie in Wikipedia-Artikel zur Verfü-

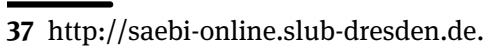

38 Vgl. Letocha, Michael: Vom ,Versuch einer Litteratur der sächsischen Geschichte‘ zur ,Sächsischen Bibliographie‘. Geschichte der landeskundlichen Bibliographien in Sachsen. In: Die Regionalbibliographie im digitalen Zeitalter. Deutschland und seine Nachbarländer. Hrsg. von Ludger Syré u. Heidrun Wiesenmüller. Frankfurt am Main: Klostermann 2006. S. 349-366; Letocha, Michael: Sächsische Bibliographie im digitalen Zeitalter. In: Neues Archiv für sächsische Geschichte 74/75 (2003/2004). S. 455-460, jeweils mit Verweisen auf die ältere Literatur.

39 Vgl. einführend Lordick, Harald: Fachspezifische und nutzerzentrierte Perspektiven Quellen vernetzen mit der Gemeinsamen Normdatei. In: Deutsch-jüdische Geschichte digital, 27. November 2016. https://djgd.hypotheses.org/1181; Lordick, Harald: BEACON - „Leuchtfeuer“ für Online-Publikationen. In: Deutsch-jüdische Geschichte digital, 17. Mai 2015. http://djgd. hypotheses.org/672. 
gung..$^{40}$ In der Bibliografie sind die GND-Normdaten mit der Pica-ProduktionsNummer (PPN) des im Südwestdeutschen Bibliotheksverbund (SWB) verwendeten Datenformats PICA verbunden. So wird zum Beispiel aus dem Quellcode \{\{SächsBib|160123801\}\} - SächsBib als Abkürzung für Sächsische Bibliografie plus die SWB-PPN zur GND-ID $118505084^{41}$ - im Bereich „Weblinks“ eines Artikels (hier: demjenigen zu Friedrich August I., genannt August der Starke) automatisiert der Verweis „Literatur von und über August II. in der Sächsischen Bibliografie“. Über den Link gelangen die Nutzer zu den mehr als 1700 Titeln, die über Sachsens bekanntesten Herrscher - in der Wikipedia mit seinem Namen als polnischer König erfasst - in der Bibliografie verzeichnet sind. ${ }^{42}$ Diese Verknüpfung war für Personendaten bereits in der ersten Version der Vorlage möglich und wurde vor allem in Artikel zu Wissenschaftlern wie dem Direktor der Königlichen Öffentlichen Bibliothek in Dresden als einer Vorgängereinrichtung der SLUB, Friedrich Adolf Ebert (1791-1834), ${ }^{43}$ oder dem Archivar am Hauptstaatsarchiv Dresden, Otto Posse (1847-1921), ${ }^{44}$ eingebunden. Eine zweite Fassung ermöglichte dann nach Rückmeldungen aus der Community die Aufnahme von Geografika wie der Massenei, ${ }^{45}$ einem geschützten Waldgebiet östlich von Dresden.

Die Nutzer des Onlinelexikons und die im landeskundlichen Bereich Beschäftigten an der SLUB können so voneinander profitieren. Mittlerweile haben Wikipedia-Nutzer die Vorlage mehr als 600-mal verwendet. Die Einarbeitung erfolgt zumeist durch die an sächsischen Themen interessierten Autoren der Enzyklopädie. Während der Arbeit an ihren Artikeln können sie die Mitarbeiter der Sächsischen Bibliografie auf fehlende Normdatensätze, nicht erfasste Publikationen oder fehlerhaft verknüpfte Artikel zu einem Thema hinweisen. ${ }^{46}$ Die Hinweise werden im Saxonica-Referat der SLUB erfasst, geprüft und systematisch abgearbeitet. So steigen Qualität und Nutzbarkeit der Wikipedia-Artikel wie der Bibliografie gleichermaßen, wird genuine fachbibliothekarische Arbeit durch bürgerschaftliches Engagement ergänzt und bereichert. Die häufige

40 https://de.wikipedia.org/wiki/Vorlage:SächsBib. Vgl. Munke, Martin: Neue Saxonica-Vorlage. Literaturverweise einfach in Wikipedia-Artikel einbinden. In: SLUBlog, 16. September 2016. https://blog.slub-dresden.de/beitrag/2016/09/16/neue-saxonica-vorlage-literaturverweise-einfach-in-wikipedia-artikel-einbinden/.

41 http://swb.bsz-bw.de/DB=2.304/PPN?PPN=160123801.

$42 \mathrm{https} / / /$ de.wikipedia.org/wiki/August_II._(Polen).

$43 \mathrm{https} / / /$ de.wikipedia.org/wiki/Friedrich_Adolf_Ebert.

44 https://de.wikipedia.org/wiki/Otto_Posse.

$45 \mathrm{https}$ ///de.wikipedia.org/wiki/Massenei.

46 Siehe die Liste auf der Nutzerseite des von den SLUB-Mitarbeitern genutzten Gemeinschaftsaccounts unter https://de.wikipedia.org/wiki/Benutzer_Diskussion:SäBi_SLUBDD. 
Nutzung der „Danken“-Funktion für eine durchgeführte Bearbeitung zeigt, dass diese Kooperationsmöglichkeit von der Wikipedia-Community gerne wahrgenommen wird. Durch die intensive Arbeit der Bibliotheksmitarbeiter in der GND können Wikipedia-Artikel noch an anderer Stelle ergänzt werden. So können die Bearbeiter neuer GND-Normdatensätze, die im Rahmen der täglichen Arbeit angelegt werden, diese in bestehende Wikipedia-Artikel einpflegen. Ein weiterer Schritt wäre die Neuanlage auch von fehlenden Artikeln in der Onlineenzyklopädie selbst, wofür im Rahmen der Diensttätigkeit allerdings keine Ressourcen zur Verfügung stehen. Weiterhin engagierten sich Mitarbeiter der SLUB aus der Abteilung Handschriften, Alte Drucke und Landeskunde bei der Kampagne „1Lib1Ref“, bei der Bibliothekare aus ihrer Kenntnis der Spezialliteratur - hier: im regional- und landeskundlichen Bereich - fehlende Quellenbelege und Literaturangaben in Wikipedia-Artikeln ergänzen. ${ }^{47}$ Über ein Onlinetool werden diese Stellen identifiziert und können nach verschiedenen Themen aufgelistet werden. Im Rahmen der Kampagne im Januar 2017 bearbeitete ein Mitarbeiter unter anderem die historischen Abschnitte in den Einträgen zu den sächsischen Industriestädten Crimmitschau ${ }^{48}$ und Hainichen. ${ }^{49}$

\section{Retrodigitalisierung und Volltextgenerierung: Digitale Sammlungen und Wikisource}

Textgrundlage für die (regionalspezifischen) Artikel in der Wikipedia ist, wie eingangs erwähnt, neben der aktuellen Forschungsliteratur noch immer die historische Grundlagenforschung, deren Ergebnisse im Rahmen der Digitalisierungsbemühungen der vergangenen Jahre vielfach online frei verfügbar vorliegen. ${ }^{50}$ Die Nutzbarkeit der Digitalisate wird signifikant erhöht, wenn neben den reinen Bilddateien ebenso durchsuchbare Volltexte vorliegen. Dies geschieht

47 Vgl. https://meta.wikimedia.org/wiki/The_Wikipedia_Library/1Lib1Ref/de. Weitere Ansätze zur systematischen Verbesserung der Qualität von Wikipedia-Artikeln seitens der Wikimedia Foundation analysieren Hübner, Qualität (wie Anm. 20); Schulenburg, Frank: Gezieltes Verbessern von Themenbereichen in der Wikipedia. Fünf Jahre Wikipedia Education Program eine Bestandsaufnahme. In: Wikipedia und Geschichtswissenschaft (wie Anm. 19). S. 93-102. https://doi.org/10.1515/9783110376357-008.

$48 \mathrm{https} / / /$ de.wikipedia.org/wiki/Crimmitschau.

49 https://de.wikipedia.org/wiki/Hainichen.

50 Vgl. als Überblick Altenhöner, Reinhard u. a.: Digitalisierung von Kulturgut. In: Praxishandbuch Bibliotheksmanagement (wie Anm. 32). S. 775-823. https://doi.org/10.1515/ 9783110303261.775. 
zum einen über automatisierte Verfahren der Optical Character Recognition (OCR), die aber gerade im Bereich der Erkennung von Frakturschriften noch mit vielen Herausforderungen zu kämpfen hat und bei der manuelle Nacharbeiten nötig sind.$^{51}$ Bei Zeitschriften treten dann Fragen der Strukturerkennung hinzu, die sich ebenfalls weiterhin als kompliziert erweisen..$^{52}$ Ein anderer Ansatz ist es daher, die Übertragungsarbeit direkt manuell vorzunehmen. Damit einher geht ein hoher personeller und mithin finanzieller Aufwand, weshalb dieser Ansatz oft nur von wenigen Enthusiasten verfolgt wird. Ein Beispiel hierfür ist das Wikipedia-Schwesterprojekt Wikisource. Die Arbeit dort kann als eine spezifische Form von Crowdsourcing, also das „Outsourcing von Teilaufgaben von Institutionen an Freiwillige, die diese Aufgaben aufgrund von Interesse und spezifischem (Fach-)Wissen in der Freizeit zu erledigen helfen" ${ }^{53}$ verstanden werden. Grundlage sind Publikationen, die bereits gemeinfrei sind oder unter einer freien Lizenz stehen. Ausgehend von digitalisierten Seiten aus Büchern oder Zeitungen werden die enthaltenen Texte transkribiert, nach dem Sechs-AugenPrinzip geprüft und korrigiert. Anschließend stehen sie im Internet frei zugänglich zur Verfügung. Die Digitalisierungsbestrebungen der großen Bibliotheken bilden eine wichtige Grundlage für die Arbeit auf Wikisource. Die mit einer entsprechenden Lizenz versehenen Digitalisate - im Fall der SLUB bisher meist CCBY 4.0, perspektivisch $\mathrm{CC}^{54}$ - werden zunächst auf dem Medienrepositorium der Wikimedia Foundation, Wikimedia Commons, ${ }^{55}$ gespeichert und anschlie-

51 Vgl. als instruktive Überblicke Springmann, Uwe, Florian Fink u. Klaus-U. Schulz: Automatic Quality Evaluation and (Semi-)Automatic Improvement of OCR Models for Historical Printings. In: ArXiv e-prints, 16. Juni 2016. https://arxiv.org/abs/1606.05157; Holley, Rose: How Good Can It Get? Analysing and Improving OCR Accuracy in Large Scale Historic Newspaper Digitisation Programs. In: D-Lib Magazine. The Magazine of Digital Library Research 15 (2009) H. 3-4. https://doi.org/10.1045/march2009-holley.

52 Vgl. Mühlberger, Günter: Digitalisierung historischer Zeitungen aus dem Blickwinkel der automatisierten Text- und Strukturerkennung (OCR). In: Zeitschrift für Bibliothekswesen und Bibliographie 58 (2011) H. 1. S. 10-18. https://doi.org/10.3196/186429501158135.

53 Graf, Nicole: Crowdsourcing - neue Möglichkeiten und Grenzen für Bildarchive. In: o-bib 1 (2014) H. 1. S. 249-253, hier S. 249. https://doi.org/10.5282/o-bib/2014H1S249-253.

54 Bonte, Achim u. Simone Georgi: Größtmögliche Offenheit. SLUB vergibt freie Lizenz für digitalisierte Objekte. In: BIS - Das Magazin der Bibliotheken in Sachsen 8 (2015) H. 1. S. 22-23. http://nbn-resolving.de/urn:nbn:de:bsz:14-qucosa-164095. Zum Konzept der Creative-Commons-Lizenzen vgl. Weller, Michael u. Elena Di Rosa: Lizenzierungsformen. In: Grundlagen der praktischen Information und Dokumentation. Handbuch zur Einführung in die Informationswissenschaft und -praxis. 6., völlig neu gefasste Ausgabe. Hrsg. von Rainer Kuhlen, Wolfgang Semar u. Dietmar Strauch. Berlin, Boston: de Gruyter 2013. S. 454-465, hier S. 460-462. https://doi.org/10.1515/9783110258264.454.

55 https://commons.wikimedia.org. Bilddaten aus dem Bestand der Deutschen Fotothek hat die SLUB ebenfalls schon früh für Wikimedia Commons zur Verfügung gestellt. Auch hier kön- 
ßend bearbeitet. Wie bei der Enzyklopädie Wikipedia ist bei den entsprechenden Projekten eine freie Mitarbeit möglich. ${ }^{56}$

Bei Wikisource-Projekten zu Themen der sächsischen Regional- und Landeskunde werden die transkribierten Volltexte auf Wunsch anschließend in die Metadaten der digitalisierten Objekte in den Digitalen Sammlungen der SLUB aufgenommen. Zwischen den Aktiven auf Wikisource und dem Saxonica-Referat der SLUB besteht entsprechend ein regelmäßiger Kontakt. Auch im Datensatz der Sächsischen Bibliografie erfolgt ein Verweis. Einerseits profitieren so die Nutzer bei Wikisource von der Arbeit der Bibliotheken, die die Datengrundlage für ihre Arbeit zur Verfügung stellen. Anderseits kann die Bibliothek ergänzende Informationen und weiterführende Angebote zu ihren Beständen in ihre Verzeichnissysteme aufnehmen. Ein aktuelles Beispiel ist ein Grundlagenwerk der sächsischen Geschichte: das fünfbändige Album der Rittergüter und Schlösser im Königreiche Sachsen aus der Mitte des 19. Jahrhunderts. ${ }^{57}$ Bereits 2004 von der SLUB digitalisiert, erfolgte, obwohl in Antiquaschrift verfasst, noch keine OCR-Behandlung der Images. Durch die Arbeit auf Wikisource liegt nun ein frei zugänglicher und elektronisch durchsuchbarer Volltext für das Werk vor. Drei Bände und das Ortsregister wurden bereits transkribiert und korrigiert, die ausstehenden Bände befinden sich in der letzten Korrekturphase. Andere für das Thema Sachsen relevante Ressourcen sind die Ausgaben des Neuen Lausitzischen Magazins der Oberlausitzischen Gesellschaft der Wissenschaften und seiner seit 1768 erschienenen Vorgängerpublikationen, für die WikisourceNutzer eine Auflistung der durch verschiedene Einrichtungen - darunter die SLUB - produzierten Digitalisate erarbeitet haben. Sie umfasst die bis 1912 erschienenen Jahrgänge. ${ }^{58}$ Ein weiteres Beispiel, bei dem der Anstoß für das Wiki-

nen Datenanreicherungen - etwa durch Georeferenzierung - zurück in die Bibliothekssysteme übertragen werden. Vgl. Bove, Jens: Open Access 2.0. Die Deutsche Fotothek der SLUB bei Wikimedia Commons. In: BIS - Das Magazin der Bibliotheken in Sachsen 2 (2009) H. 1. S. 23-24. http://nbn-resolving.de/urn:nbn:de:bsz:14-ds-1237556606026-59807.

$56 \mathrm{Vgl}$. Bemme, Jens: Eisberg voraus! Mit Wikisource historische Texte einfach in offene Quellen verwandeln. In: SLUBlog, 3. November 2016. https://blog.slub-dresden.de/beitrag/ 2016/11/03/eisberg-voraus-mit-wikisource-historische-texte-einfach-in-offene-quellen-verwandeln/.

57 https://de.wikisource.org/wiki/Album_der_Rittergüter_und_Schlösser_im_Königreiche_Sachsen. Zum von Gustav Adolf Poenicke (1807-1867) herausgegebenen Werk vgl. Täuber, Ines: Der „Poenicke“. Eine Präsentation sächsischer Rittergüter. In: Der Schritt in die Moderne. Sächsischer Adel zwischen 1763 und 1918. Hrsg. von Silke Marburg u. Josef Matzerath. Köln, Weimar, Wien: Böhlau 2001. S. 95-114.

58 https://de.wikisource.org/wiki/Neues_Lausitzisches_Magazin. Vgl. im Zusammenhang Lemper, Ernst-Heinz: Zur Geschichte und Bedeutung der Oberlausitzischen Gesellschaft der Wissenschaften zu Görlitz. In: Neues Archiv für Sächsische Geschichte 64 (1993). S. 85-95. 
source-Projekt durch einen Mitarbeiter der SLUB erfolgte, ist die Transkription des Jahrbuchs der deutschen Radfahrer-Vereine 1897/98. ${ }^{59}$ Für spezifische Forschungsfragen besonders zu "Nischenthemen“ wie der historischen Radfahrerliteratur schaffen solche Projekte Sichtbarkeit und Anschlussfähigkeit für vergleichbare Vorhaben.

\section{Communitys im Austausch: Wikipedia- Sprechstunden und -Stammtische, WikiCon und WikiLibrary Barcamp}

Jenseits der gemeinsamen Arbeit im virtuellen Raum ist auch der direkte Austausch wichtig für die Identifizierung von Ansatzpunkten für eine Kooperation, das Vorantreiben von Projekten und schließlich für den Abbau von Ängsten und Hürden, wie sie nicht nur zwischen Wiki-Communitys und Wissenschaft, sondern ebenso zwischen Wiki-Communitys und (wissenschaftlichen) Bibliotheken noch immer existieren. In unregelmäßigen Abständen findet daher an der SLUB seit April 2016 eine Wikipedia-Sprechstunde statt, in der Wikipedianer mit Bibliotheksnutzern und -mitarbeitern ins Gespräch kommen. ${ }^{60}$ Themen waren bisher unter anderem eine allgemeine Einführung zur Arbeit in der Onlineenzyklopädie und eine Vorstellung des Schwesterprojekts Wikisource. Außerdem traf sich der Stammtisch der Wikipedianer aus Dresden und Umgebung bereits mehrfach in der SLUB, zuletzt im Mai 2017. ${ }^{61}$ Zur Vorstellung der eigenen Angebote und zum Ausloten weiterer Berührungspunkte waren auf dem letzten Jahrestreffen der Wiki-Communitys, der WikiCon vom 8. bis 10. September 2017 in Leipzig, ${ }^{62}$ zwei Mitarbeiter aus dem Saxonica-Referat der Bibliothek vertreten. Sie präsentierten und diskutierten zu den Themen „Zusammen.Spiel.Plätze. Dreiklang Citizen Science: Wikipedia, Wikisource und Bibliothek“ und „Neue alte Sammelgebiete. Werkzeuge für kollaborative Informations- und Quellensammlungen in wiederentdeckten (historischen) Themenfeldern“.

59 https://de.wikisource.org/wiki/Jahrbuch_der_deutschen_Radfahrer-Vereine.

60 Vgl. Stöhr, Matti: Sprechstunde: So funktioniert Wikipedia. In der SLUB am 27. April. In: SLUBlog, 26. April 2016. https://blog.slub-dresden.de/beitrag/2016/04/26/sprechstunde-sofunktioniert-wikipedia-in-der-slub-am-27-april/.

61 Vgl. Bemme, Jens: Tech on Tour. Wikipedia-Stammtisch am 30. Mai 2017. In: SLUBlog, 29. Mai 2017. https://blog.slub-dresden.de/beitrag/2017/05/29/tech-on-tour-wikipediastammtisch-am-30-mai-2017/.

62 https://de.wikipedia.org/wiki/Wikipedia:WikiCon_2017. 
Bisheriger Höhepunkt des gegenseitigen Austausches war ein knappes Jahr zuvor das von der Wikimedia Foundation, dem „Netzwerk Bibliothek“ des Deutschen Bibliotheksverbandes und der SLUB veranstaltete erste WikiLibrary Barcamp am 3. Dezember 2016 in Dresden. ${ }^{63}$ Mehr als 60 Teilnehmer diskutierten in fast 20 Sessions über Berührungspunkte zwischen Wiki- und Bibliothekswelten, über gemeinsame Instrumente und Gesprächsformate. Wie in Barcamps üblich, gab es im Vorfeld kein festgelegtes Programm. Stattdessen stimmte das Publikum über die Durchführung der in einminütigen Kurzimpulsen vorgestellten Themen $a b .{ }^{64}$ Ausgangspunkt der Debatten war die Feststellung, dass die Wikipedia in den vergangenen 15 Jahren ,anscheinend zu einem der großen Wegweiser im Land der wissenschaftlichen Information geworden [ist]“65 - eine Rolle, die traditionell den Bibliotheken zugeschrieben wurde und die nun aufgerufen sind, sich mit den Veränderungsprozessen der digitalen Transformation auseinanderzusetzen. Führen doch diese Prozesse dazu, dass Bibliotheken nur mehr ein Anbieter in einer Welt sind, die Wissen zunehmend frei(er) verfügbar macht - unter anderem durch Projekte wie die Wikipedia. Dass es dabei sinnvoller ist, miteinander zu kooperieren anstatt zu konkurrieren, war Konsens der Teilnehmer.

Konkreter Ansatzpunkt für eine Zusammenarbeit ist z. B. die freie Datenbank Wikidata. ${ }^{66}$ Bibliotheken wie die SLUB nutzen die Plattform zunehmend dafür, eigene Daten zu organisieren und anzureichern. Umgekehrt können diese Daten dann von anderen nachgenutzt werden. Die Bereitbestellung allein reicht allerdings meist nicht aus, denn Informationsarbeit ist nötig, um die Verwendungsmöglichkeiten bekannt zu machen. Es braucht also Personen, die in beiden Welten unterwegs sind, um die Potenziale einer Kooperation wirklich zu nutzen. ${ }^{67}$ Gleichzeitig hängt die Wahrnehmung der zahlreichen Berührungs-

63 https://de.wikipedia.org/wiki/Wikipedia:WikiLibrary_Barcamp_2016/WikiLibrary_Barcamp_2016.

64 Vgl. Fischer, Barbara: Aus Ideen werden Projekte werden Ergebnisse werden Ideen. In: Wikimedia Deutschland Blog, 8. Dezember 2016. https://blog.wikimedia.de/2016/12/08/ausideen-werden-projekte-werden-ergebnisse-werden-ideen/. Die während des Barcamps geführten Etherpads und die Begleitung via Twitter sind auszugsweise dokumentiert bei Bemme, Jens: Bibliotheken im Netz. Rückschau auf das WikiLibrary Barcamp. In: SLUBlog, 4. Dezember 2016. https://blog.slub-dresden.de/beitrag/2016/12/04/bibliotheken-im-netz-rueckschau-aufdas-wikilibrary-barcamp.

65 Heller, Ozean des freien Wissens (wie Anm. 15).

66 https://www.wikidata.org.

67 Vgl. Heller, Lambert u. Tracy Hoffmann: Aus Ideen werden Projekte, werden Ergebnisse, werden Ideen. Das bundesweit erste WikiLibrary Barcamp 2016. In: BIS - Das Magazin der Bibliotheken in Sachsen 10 (2017) H. 1. S. 28-31, hier S. 31. http://nbn-resolving.de/urn:nbn: de:bsz:14-qucosa2-79356. 
punkte zwischen wissenschaftlichen Bibliotheken und Wiki-Communitys bisher allzu häufig von diesen engagierten Einzelpersonen und individuellen Initiativen ab. Bis zu einer Verstetigung scheint es noch ein weiter Weg zu sein. Seitens der Bibliotheken ist es wichtig, kollaborative Arbeitsformen im Sinn des Citizen-Science-Gedankens weiter zu stärken und zu unterstützen. An der SLUB geschieht dies künftig unter anderem mit dem neuen Landeskundeportal „Saxorum “, 68 das solche Arbeitsformen etwa mit der Möglichkeit, den Datenbestand der Sächsischen Bibliografie zu ergänzen, institutionalisieren soll - ein Schritt zu einer vernetzten digitalen Landeskunde in und für Sachsen.

68 Vgl. Munke, Martin: Saxorum. Neues Landeskundeportal für Sachsen. In: BIS - Das Magazin der Bibliotheken in Sachsen 10 (2017) H. 3. S. 154-157. http://nbn-resolving.de/urn: nbn:de:bsz:14-qucosa2-169724; Munke, Martin: Neue Impulse für eine digitale Landeskunde in Sachsen. Die Onlineportale Sachsen.digital und Saxorum. In: Sächsische Heimatblätter 64 (2018) H. 1, S. 72-77. 